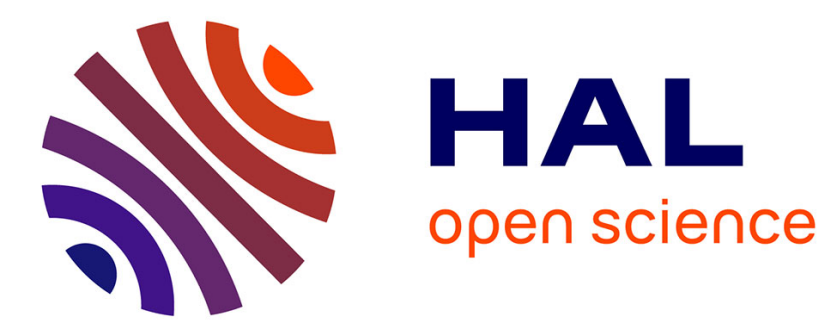

\title{
Viande et questionnaires:de nouvelles sources pour une nouvelle écriture de l'histoire
}

\author{
Andrea Petö
}

\section{To cite this version:}

Andrea Petö. Viande et questionnaires:de nouvelles sources pour une nouvelle écriture de l'histoire. Archives et histories dans les sociétés post-communistes ed. Sonia Comb, 2009. hal-03220059

\section{HAL Id: hal-03220059 \\ https://hal.science/hal-03220059}

Submitted on 6 May 2021

HAL is a multi-disciplinary open access archive for the deposit and dissemination of scientific research documents, whether they are published or not. The documents may come from teaching and research institutions in France or abroad, or from public or private research centers.
L'archive ouverte pluridisciplinaire HAL, est destinée au dépôt et à la diffusion de documents scientifiques de niveau recherche, publiés ou non, émanant des établissements d'enseignement et de recherche français ou étrangers, des laboratoires publics ou privés. 
problèmes d'approvisionnement ont par ailleurs causé de nombreuses tensions au sein même de la société. L'analyse détaillée de cette question aux facettes multiples ne serait pas uniquement un défi intellectuel intéressant, elle permettrait surtout d'éclairer la question du fonctionnement au quotidien du système institutionnel de ce qu'on appelle la "République populaire de Pologne ".
Viande et questionnaires :

de nouvelles sources

pour une nouvelle écriture de l'histoire ${ }^{1}$

Andrea Petó

\section{VIANDE ET RÉSISTANCE}

Les deux textes précédents représentent deux approches exploitant les nouvelles sources de recherches historiques, disponibles depuis 1990. Je voudrais me focaliser sur un lien en particulier : les différentes formes de pouvoir disciplinaire et les moyens d'utiliser les sources historiques de façon critique pour historiciser les relations de pouvoir. L'article de Darius Jarosz porte sur l'histoire politique de la viande. L'étude de l'alimentation est devenue un thème de recherche important. Elle met en exergue la dimension sociale de la nourriture et possède un grand potentiel analytique ${ }^{2}$. Je vais mentionner ici quatre points qui peuvent donner lieu à une étude approfondie.

En premier lieu, la production, la préparation, la consommation, l'attitude et la symbolique de la nourriture s'inscrivent dans le temps et dans l'espace, qui sont définis selon le sexe, la classe sociale, le groupe ethnique, les croyances et le contexte culturel.

En second lieu, l'analyse du discours alimentaire met en lumière les processus impliqués par la question de l'identité selon les différents systèmes alimentaires traditionnels dans différents contextes politiques.

Le discours alimentaire possède plusieurs fonctions sociales et encode les événements sociaux. Parler de la nourriture (de sa préparation et de sa consommation) sert de marqueur identitaire et de cadre aux

1. Traduit de l'anglais par Irina Goriounov et Olivier Ouzilou

2. Voir un résumé de la documentation dans A. Petö, « Food-talk. Markers of Identity and Imaginary Belongings " dans Women Migrants from East to West. Gender, mobility and belonging in contemporary Europe, L. Passerini, D. Lyon, E. Capussoti, I Laliotou (dir.), Oxford-New York, Berghann Books, 2007, p 152-165. [uLe discours alimentaire. Marqueurs d'identité et appartenance imaginaire " dans Les Femmes migrant d'Est en Ouest. Sexe, mobilité et appartenance dans l'Europe contemporaine.] 
formes de narration de la différence et de la ressemblance. Il s'agit d'un métadiscours : tout le monde connaît et, apparemment, tout le monde comprend la nourriture, c'est-à-dire le fait qu'il faut manger pour surcomprend la nourriture, c'est-à-dire "facile " à utiliser lorsque des questions importantes sont abordées.

Quatrièmement, le discours sur l'alimentation est également un Quatre permettant de construire une appartenance imaginaire. Ce discours, grâce à sa flexibilité en matière de sujets et d'émotions, sert en effet de lieu pour la construction de soi et de l'autre.

Enfin, et c'est là le cinquième élément, la nourriture a des fonctions Enfin, et c'est là le cinquième elément, la nù les femmes ont pour rôle productives et regulatrices. Dans la mesure où les fenton onsomme cette admis la preparation de la nourriture et qu'ensuite on consomme rituels, les nourriture en tant que membre de la communates a nourriture entraînent des formes de résistance et d'oppression.

Dans son étude, Dariusz Jarosz décrit l'histoire de la Pologne du

Dans son étude, Dources sont soipoint de vue de la consommation de la viande. Ses soures statistique disant des sources historiques traditionnelles. Les données statistiques ne sont pas très fiables à cause de la manie qu'avait l'économie planifiée de vouloir contrôler les chiffres, ce qui a entraîné une inflation des sources. Par le passé, j'ai réalisé une enquête sur l'évolution des donsources. Par le passe, $\mathrm{j}$ ai réalise le lévénement, à travers le nées statistiques en partant du lieu de levénement, à travers le Département régional du bureau des statistiques, jusqu'à ce qu'elles atterrissent sur le bureau du chef du Département de la propagande au Comité central. Ces données rendaient compte non pas de la réalité Comite central. Ces doprésentations. Je suggère donc de les considérer mais d'idées et de représentations. Je suggère done de les considerer comme des indicateurs des relations de pouvoir au lieu de les voir comme de vraies données statistiques : la logique qui est à leur

présente plus d'intérêt que le potentiel explicatif des chiffres.

Cependant, dans son article, Dariusz Jarosz utilise egalement d autres sources : des sources orales, les documents du Parti communiste et, de façon très innovante, les entretiens qu'il a réalisés auprès de personnes pouvant témoigner de leur expérience des files d'attente pour acheter de pouvant temoigner de leur experiéc des histoires drôles et des anecdotes la viande. Il a egalement rassem. Je voudrais retenir une idée de tout son en rapport avec l'alimentation. Je voudrais retenir the idee dout son travail remarquablement riche : la question de la discipline du pouvoir, que je voudrais relier au problème théorique de la façon dont on peut anaque je voudrais relier au piobire de l'oppression. Comme je l'ai dit dans - P. Andrea, « Lebensumstaenđe der Arbeiter in einem Grosswerk in den 1950er 3. P. Andrea, « Lebensumstaende der Arbelter thandes usines des années 1950) dant Jahren (Conditions de vie des travaillium Hungaricae, Budapest, vol. 35. 1989 (1-4), Acta Historica p. $251-265$ l'introduction, le discours alimentaire a un potentiel heuristique : celui de permettre aux historiens de la société de parler des différentes relations du pouvoir et de démêler la texture très complexe du fonctionnement du pouvoir dans un certain contexte historique.

Dariusz Jarosz a mentionné dans son étude le fait que le pouvoir a tenté de substituer à la consommation de viande celle du lait en faisant appel à des arguments de nature diététique. On oublie souvent que parmi les objectifs du projet manqué de gauche en Europe de l'Est, il y avait celui de simplifier la production agroalimentaire, de libérer la femme de sa cuisine et également d'introduire d'autres valeurs associées à l'alimentation dans une Europe de l'Est pauvre et profondément sousalimentée $e^{4}$ La viande symbolisant l'abondance, une abondance malsaine comme nous le savons aujourd'hui, il est évident que l'expérience communiste a eu des conséquences controversées.

Parmi ces dérives, on trouve le fait que le Parti communiste se servait du système des privilèges et des privations comme d'un instrument disciplinaire. Dariusz Jarosz établit également un lien entre la pénurie de viande et la mobilisation sociale en Pologne. Je voudrais cependant faire remarquer qu'il existait d'autres formes de résistance qui remettaient en question le rôle distributif du Parti communiste, comme les histoires drôles : l'《 arme des faibles », pour citer l'œuvrc de James Scott, n'était pas uniquement une plaisanterie. Ces dissidents polonais, qui refusaient de faire la queue pour de la viande, sont devenus des végétariens. Ils ont composé un livre de recettes que j'utilise encore aujourd'hui car les ingrédients nécessaires ne sont pas des ingrédients de la cuisine asiatique ou d'Extrême-Orient mais des légumes « ordinaires ». Pour aller plus loin, il ne faudrait pas seulement réaliser une analyse détaillée du fonctionnement des systèmes du pouvoir sur la base d'une division qui oppose ceux qui veulent de la viande aux dirigeants qui ne la donnent pas, c'est-à-dire « eux » à « nous ». Il faudrait également analyser l'influence de ces systèmes du pouvoir sur les relations entre les deux sexes et les formes de résistance selon le sexe en Pologne, au-delà du contexte politique communiste.

L'article de Dariusz Jarosz contribue à développer une autre perspective offerte par l'analyse des fonctions régulatrices de l'alimentation. Il utilise la consommation pour analyser l'histoire de la Pologne, alors que jusqu'à aujourd'hui les historiens considéraient que l'histoire échappait à l'empire de la consommation. Ses travaux nous

4. H. Rothstein, R. A. Rothstein, «The Beginning of Soviet Culinary Arts in Glants », M. Toomre (dir.) Food in Russian History and Culture, Bloomington, Indiana University Press, 1997, p. 177-195. 
apprennent que la non-consommation, la rareté des biens, ne sont pas apprent reléguées hors des frontières de mesure our a les l'histoire mais qu'elles sont un discours dans la mesure où " les discours... (sont) des pratiques qui forment systématiquement les objets dont ils parlent ${ }^{5} »$. La consommation est marquée par une distinction des sexes et les stéréotypes qui reprennent cette distinction depuis bien longtemps. Ils ont survécu à l'époque du manque, au communisme et se portent très bien dans notre capitalisme de marché mondial. Mais, si la contestation est possible en période de manque, elle devrait également être présente en période d'abondance. Depuis la chute du communisme, la consommation est considérée comme la seule mesure de la valeur par des millions de personnes qui en sont exclues, alors il faudrait penser à d'autres formes de contestation. L'héritage du communisme a donc toujours des choses à nous apprendre.

\section{ARCHIVAGE STALINIEN, HISTOIRE POSTSOVIÉTIQUE :}

LA MARGINALISATION EN QUESTTON

Dans un contexte assez sensiblement différent, Sergeï Krasilnikov recourt à une quantité considérable de nouvelles archives pour retracer l'histoire sociale de la Sibérie occidentale. Cependant la logique de conservation minutieuse et de constitution des dossiers de l'Union soviétique stalinienne influence la façon dont on écrit sur cette période. Se concentrer sur les soi-disant " groupes marginaux » conduit à renforcer la logique du système stalinien. En les identifiant comme "marginaux", on enferme déjà ces hommes et ces femmes dans le « marginax $n$, on enferme dejà ces hommes et ces femmes dans le contexte stalinien. Le pouvoir disciplinaire utilise la politique de transfert de populations afin de contrôler certains groupes sociaux. Ces groupes sont nommés et créés par la logique du pouvoir disciplinaire. En conceptualisant simplement le pouvoir de manière dichotomique, la tentation est trop forte pour les historiens d'utiliser des concepts tout faits, sans réflexion critique. Or la vie de ces groupes sociaux ne devrait pas être représentée à partir de cette logique.

J'aimerais dialoguer avec l'auteur pour connaître la manière dont il utilise le terme d' « adaptation " dans son article. Particulièrement, lorsqu'il mesure la capacité d'《 adaptation » des victimes des transferts staliniens au nombre d'enfants nés dans une société où aucun autre moyen de contrôle de la reproduction autre que l'avortement n'était disponible. Je propose d'y substituer les concepts analytiques plus

\footnotetext{
5. M. Foucault, L'Archéologie du Savoir, Paris, Gallimard, 1969, p. 66-67.
}

féconds d'exclusion et d'inclusion - et pas seulement parce que ces concepts ouvrent un espace pour une compréhension dynamique des différentes manières dont les groupes changeaient de statut selon le type de relations qu'ils entretenaient avec le pouvoir.

Cette approche analytique reconnaît l'existence de différents groupes et prend en compte les différentes formes de subjectivité et d'intersubjectivité personnelle et collective. L'expression « exclusion sociale " entendue comme une pratique de pouvoir disciplinaire et d'ingénierie de construction sociale nous en dit précisément beaucoup plus sur ces processus.

L'administration soviétique était connue pour faire du recueil des données une fin en soi, ce qui était aussi une forme de contrôle des citoyens. Krasnilkov utilise ces dossiers malgré le fait que l'utilisation de questionnaires pré-remplis comporte de nombreux dangers méthodologiques. Des précédentes recherches sur les récits de vie et l'étude des curriculum vitae de cadres pendant la période du communisme m'ont démontré ce danger. Les mêmes personnes produisaient des récits de vie très différents selon la manière dont le contenu des catégories statistiques était, au hasard, modifié. Et ils transformaient leurs propres vies conformément à ce qu'ils imaginaient être les attentes du pouvoir.

Krasnilkov suppose que les familles paysannes normales avaient à leur tête des hommes. Une réflexion plus approfondie sur la définition de la normalité est ici requise. Des données intéressantes montrent qu'en dépit du projet émancipateur de gauche qui voulait changer les relations homme/femme via les statistiques, c'est l'ordre patriarcal qui a été renforcé à travers le pouvoir disciplinaire. Le concept de normalité peut signifier différentes choses. Je l'ai appris de la recherche d'un de mes étudiants qui interviewait des générations de femmes coréennes déportées en Kirghizie. La structure familiale de cette partie de l'Union soviétique ressemble à ce qui est décrit dans cet article : la plupart des femmes étaient à la tête des ménages. C'est un phénomène également bien connu en Pologne : les hommes étaient emmenés dans le cadre des politiques de transfert et les femmes restaient à la maison avec les enfants et les personnes âgées. Cela signifie-t-il qu'il s'agissait de groupes marginaux ? Pas du tout ! Notre tâche en tant qu'historien n'est pas seulement de dépasser les problèmes de conceptualisation émanant des sources sur lesquels nous travaillons, mais également de réfléchir à la manière dont les relations de pouvoir fonctionnent. Ce n'est pas simple, mais pas impossible non plus. 
COMMENTATRE FINAL COMPARATIF

Ces deux articles touchent implicitement à une question importante: la transformation des relations entre les sexes durant le communisme. Le projet de construction sociale du communisme a démantelé de manière effective et brutale les anciennes structures familiales. Pendant manière effective et brutale les anciennes structures familiales. Pendant groupes sociaux déportés de Sibérie, à des défis inattendus. Face à ces défis, certains étaient mieux équipés que d'autres.

Les auteurs de ces deux articles ne commettent pas l'erreur de réfléchir dans le cadre du dualisme simpliste opposant la victime au bourreau et ils gardent également leur distance face aux généralisations, comme celle qui consiste à dire que « les femmes » devaient affronter les dures circonstances économiques et sociales. Mais ce qui est clair, à partir de leur travail, et qu'on pourrait en fin de compte retenir, c'est que ce sont les différences de genres qui donnent des clés pour cerner des caractéristiques du communisme. Toutefois, un élément n'est pas pris en compte : le fait que, quoique les femmes - de façon intentionnelle ou non - aient obtenu du pouvoir, elles restèrent éloignées des centres de décision politiques. Si nous considérons la période post-1990, nous constatons que la tendance est tristement similaire.

Les deux auteurs utilisent des sources non orthodoxes pour leur travail historique, comme les plaisanteries et des questionnaires indisponibles auparavant pour mener des enquêtes. Ils ont tous deux tenté d'éviter les œillères de l'histoire politique de l'Europe de l'Est. C'est une entreprise dont nous devrions tous bénéficier à long terme.
13

L'ouverture des archives d'une police politique communiste: le cas tchèque, de Zdena Salivarová à Milan Kundera

Muriel Blaive ${ }^{1}$

"II suffit qu'un régime policier se maintienne vingt ans au pouvoir pour faire de tous ses complices. Même đe ses victimes m.

Jan Zábrana, Toute une vie.

Lorsque l'on considère un régime dictatorial passé où le secret jouait un rôle important, l'ouverture des archives est la pré-condition indispensable à un débat de société qui permette aux citoyens de régler leurs comptes avec un minimum de fair-play, que ce soit entre eux ou avec leur propre conscience. Mais ce n'est pas une condition suffisante et le timing n'en est pas négligeable: l'ouverture progressive des archives de l'ancienne Sécurité d'État tchécoslovaque (Státn bezpečnost ou StB) montre en effet à quel point il est devenu difficile d'assainir une atmosphère sociale empoisonnée par deux décennies de réflexion collective désordonnée et négligente d'autrui, bien souvent empreintes de méchanceté et de mesquinerie et marquées par des scandales médiatiques à répétition.

L'état morose du débat face au passé communiste en République tchèque s'explique sans aucun doute à l'origine par le fait que, à l'instar des autres pays ex-socialistes et pour les mêmes raisons tenant à un sentiment diffus de culpabilité métaphysique par rapport à sa propre compromission avec l'ancien régime ${ }^{2}$, la Tchécoslovaquie puis République tchèque a choisi après 1989 une politique $\mathrm{d}^{\prime}$ 'in-justice

1. Je youdrais adresser un remerciement tout particulier à Lubor Jílek, ainsi qu'à Sonia Combe, Sandrine Kott, Thomas Lindenberger, Paul Gradvohl et Nicolas Maslowski pour leurs remarques et commentaires critiques sur ce texte.

2. L'expression " culpabilité métaphysique ", bien que formulée dans le contexte quelque peu diffërent de la période de " normalisation » (1969-1989) est de Jan Zábrana dans Toute une vie, Allia, Paris, 2006, p. 36. 